\title{
Step like surface potential on few layered graphene oxide
}

\author{
M. Jaafar, G. López-Polín, C. Gómez-Navarro, ${ }^{\text {a) }}$ and J. Gómez-Herrero \\ Dpto. Física de la Materia Condensada, Universidad Autónoma de Madrid, 28049 Madrid, Spain
}

(Received 3 October 2012; accepted 4 December 2012; published online 28 December 2012)

\begin{abstract}
We report surface potential maps of few layered graphene oxide films on different substrates. Kelvin probe force microscopy images reveal that the surface potential decreases in steps with increasing number of layers on the substrate until five layers are reached, where it saturates to a constant value. This intrinsic behavior is smeared out in the presence of ambient humidity where the surface potential is shielded by the presence of a thin water layer on the surface. This effect can be exploited to quickly determine the number of layers of graphene oxide on a substrate. (C) 2012 American Institute of Physics. [http://dx.doi.org/10.1063/1.4773357]
\end{abstract}

Recent advances in graphene production and the extraordinary electronic, ${ }^{1}$ mechanical, ${ }^{2}$ and thermal ${ }^{3}$ properties exhibited by this unusual material have made graphene the most recent endeavour for applications of carbon based materials in current technology. Its two dimensionalities make it compatible with existing planar technology; therefore, most of the proposed applications involve the use of few layered thin films. At these very low coverage, interaction with the substrate has been proved to be critical; indeed, it has been shown that properties such as charge carrier mobility, ${ }^{4}$ doping level, ${ }^{5}$ or hydrophobicity ${ }^{6}$ differ from the bulk value and depend on the substrate and number of layers studied.

Among other routes for the production of graphene, such as mechanical exfoliation of graphite, ${ }^{7}$ chemical vapor deposition $^{8}$ on metals or silicon carbide graphitization, ${ }^{9}$ the chemical route based on the oxidation of graphite, and subsequent reduction of graphene oxide $(\mathrm{GO})^{10}$ has important advantages; its scalability, low price, and high yield of monolayers (ML) makes the layers such obtained highly attractive for assembly of structures on different substrates by cheap solution processes. The result of oxidising graphite, usually known as GO, are graphene layers decorated with epoxy and hydroxyl groups ${ }^{11}$ making these layers highly hydrophilic and water soluble. Although GO layers are electrically insulating, chemical reduction yields a partial recovery of the initial conductivity of graphite ${ }^{12,13}$ making GO and its reduced derivative ( $\mathrm{rGO}$ ) suitable for many applications that include transparent conducting films, ${ }^{14}$ sensors, ${ }^{15}$ double layered capacitors, and electrochemistry based devices. ${ }^{16}$ For many of these applications, the interaction of GO with the supporting substrate and with adjacent layers plays a relevant role in properties such as adhesion, charge transfer, doping level, etc.

Here, we focus on the surface potential behaviour of graphene sheets obtained via chemical reduction of graphite oxide on metallic type surfaces. Our results give insights into the charge exchange and the spatial distribution of charge carriers in the GO-GO and GO-substrate interfaces. Surface potential maps on three different substrates ( $\mathrm{Au}$, highly ordered pyrolytic graphite (HOPG) and highly p-doped $\mathrm{Si}$ )

\footnotetext{
${ }^{\text {a) }}$ Author to whom correspondence should be addressed. Electronic mail: cristina.gomez@uam.es.
}

reveal variations of the surface potential as a function of the number of layers deposited on the substrate; for few layers, the surface potential decreases in steps as the number of layers increases and for coverages above five layers, this behaviour breaks down and the surface potential reaches a constant value. In contrast to pristine graphene, ${ }^{17,18}$ we observe that the high hydrophobicity of these films causes that for ambient humidity above $\sim 30 \%$ this intrinsic behaviour is smeared out by water shielding of the surface potential. Finally, we demonstrate that this effect can be exploited for a fast and non-invasive unequivocal determination of the number of GO layers on different substrates, which, as shown here, will be critical for device operation.

GO flakes obtained from NanoInnova Technologies, ${ }^{19}$ oxidized by Hummer method, ${ }^{20}$ were adsorbed on $\mathrm{Au}$, HOPG, and highly p-boron doped Si (111) (see supplementary information (SI) for a detailed description). ${ }^{30}$ The samples so prepared were characterized by atomic force microscopy ${ }^{21}$ (AFM) to optimize the density of layers on the surface (we choose $\sim 50 \%$ coverage as a convenient figure for the experiments). In order to access the intrinsic properties of the surface avoiding the possible presence of adsorbates and/or a thin layer of water, if not stated different, all the measurements shown here were performed in high vacuum conditions (pressure $10^{-5}$ mbar) and a sample temperature of $60^{\circ} \mathrm{C}$. Using an $\mathrm{AFM}$ in vacuum has the added value of improving sensitivity. ${ }^{22}$ For stability and simplicity, images in vacuum environment were acquired in drive amplitude modulation mode. ${ }^{23}$ Typical AFM topographic images of our samples showed flakes with lateral dimensions ranging from 5 to $50 \mu \mathrm{m}$ and heights of $1 \pm 0.2 \mathrm{~nm}$, indicative of single layers. Although with this technique the majority of the material obtained are single layers of GO, most of the large layers $\left(>50 \mu \mathrm{m}^{2}\right)$ adsorbed on the surface presented overlapping regions; this allowed us to easily perform thickness dependent studies. On these samples, we carried out simultaneous Kelvin probe force microscopy (KPFM) measurements to obtain surface potential maps. KPFM is currently a well established technique to characterize local surface potential at the nanoscale ${ }^{24}$ where the tip-sample electrostatic interaction is minimized by the application of an appropriated combination of $\mathrm{dc}$ and ac bias voltage during topographic imaging (technical details can be found in SI). 
Figure 1(a) displays an AFM topographic image of GO flakes on a gold substrate and panel (b) corresponds to the surface potential map acquired simultaneously. Already at first sight, we can appreciate several well distinguished equipotential areas, i.e., the brightest region corresponds to the Au substrate and the darker ones correspond to areas covered by GO. A representative surface potential profile can be found in panel (c) (surface potential of the substrate was established as zero, for reference). Panels (d), (e), (g), and (h) depict topographic and surface potential maps on Si and HOPG surfaces. On the three substrates, we observe equipotential zones that by thorough inspection of the topographic images, we can correlate with areas with different GO coverage. A quick analysis of the KPFM maps shows that the surface potential difference between the 3 substrates and the first GO layer is between 100 and $200 \mathrm{mV}$ while surface potential steps between different layers are few tens of $\mathrm{mV}$. Further statistical analysis of KPFM images of GO layers on $\mathrm{Au}, \mathrm{HOPG}$, and $\mathrm{Si}$ with coexisting low and high coverage reveals that the drop of surface potential saturates as the number of layers is increased, reaching a constant value above 5-7 layers. This is also illustrated in panels (d)-(f) of Figure 1; panel (d) shows a topographic image of $\mathrm{GO}$ on a $\mathrm{Si}$ surface where identification of the number of layers by topographic means is straight-forward. In this region, we find areas with coverages of $1,2,3,5,7$, and 9 monolayers by superposition of different GO flakes. Figure 1(e) displays the corresponding KPFM image of the same region, where areas with different coverages can be clearly distinguished. The profile depicted in panel (f) quantifies the difference in surface potential from layer to layer. While clear steps of several tens of millivolts can be measured for less than 5 layers coverage; for more than 5 layers, this behavior saturates and the surface potential reaches a constant value independent of the number of layers. A quantitative summary of our results is depicted in panel (j) of Figure 1. Surprisingly, we observe that the difference in surface potential between the substrate and the first GO layers and the difference between layers are more sensitive to the level of oxidation of the layers than to the type of substrate (see Fig. S1 in supplementary information for more details).

The reduced form of the described layers was studied subsequently. Once deposited on the substrates, and characterized as explained above, GO layers were chemically reduced by thermal annealing of the substrates in high vacuum conditions for $1 \mathrm{~h}$ at $420^{\circ} \mathrm{C}$. These reducing conditions lead to a conductivity improvement of the layers of more than three orders of magnitude. ${ }^{25}$ Comparison of surface potential maps of the samples, acquired on the same area before and after chemical reduction (see Figure 2), reveals a similar behavior of the rGO films as for the GO ones. As main differences, we found lower steps in surface potential
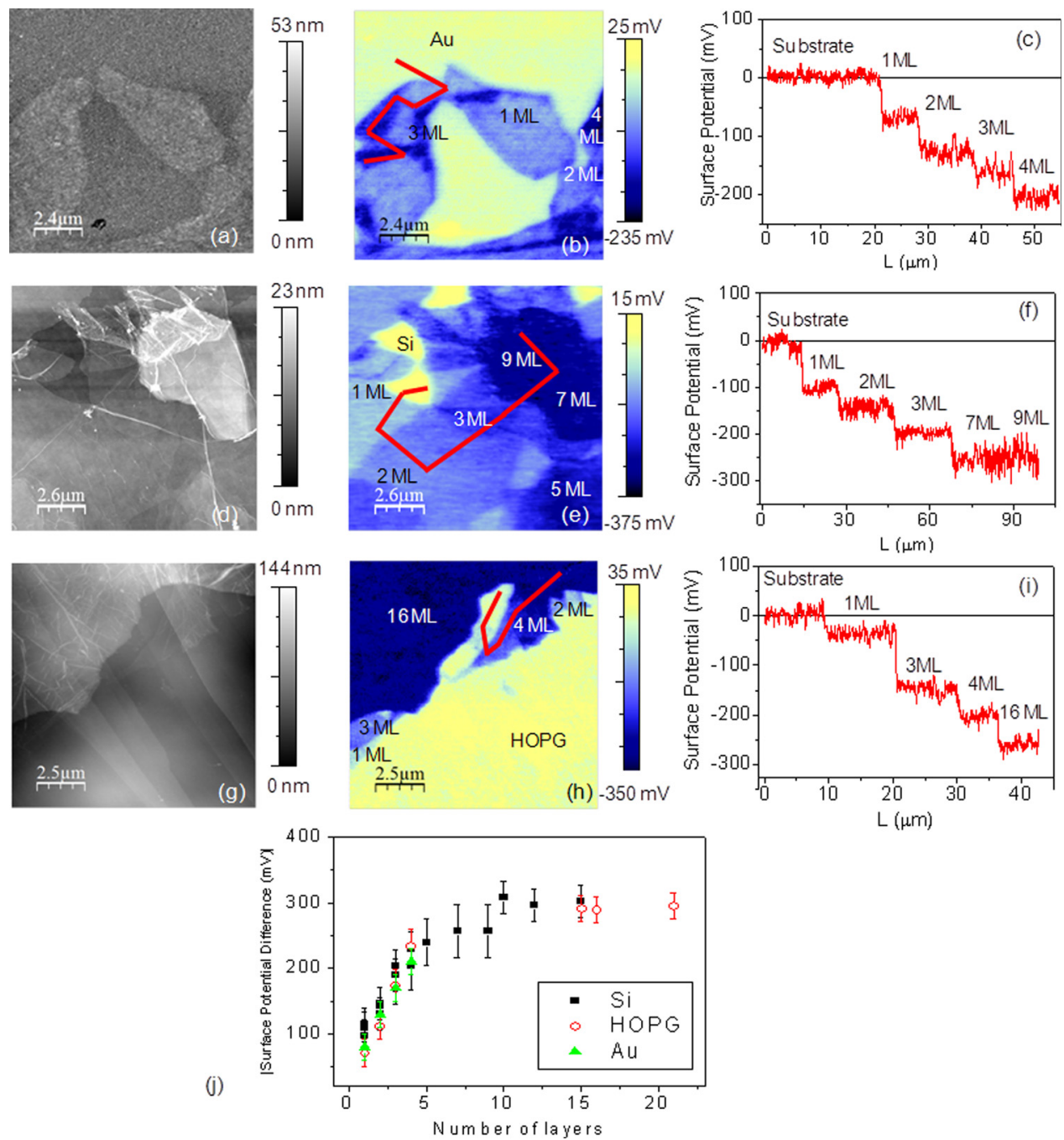

FIG. 1. (a) AFM topographic image of GO layers on a gold substrate. Panel (b) depicts a surface potential map of the same region where equipotential areas corresponding to different coverages can be appreciated. Coverage is marked with the number of ML on the substrates as $n$ ML. (c) Profile performed on the surface potential map following regions with different coverages (The red line in (b) indicates schematically the paths followed to perform the profile). (d) AFM topography image and (e) surface potential map of GO layers on a highly $p$ doped Silicon substrate. (f) Profile performed on (e) following regions with different coverage (the red line in (e) indicates schematically the paths followed to perform the profile). (g) AFM topography image and (h) surface potential map of GO layers on a HOPG substrate. (i) Profile performed on (h) following regions with different coverages (the red line in $(h)$ indicates schematically the paths followed to perform the profile). (j) This graph summarizes our experimental results: plot of the surface potential difference (in absolute value) as a function of the number of monolayers layers of GO flakes on $\mathrm{Si}$ (black), HOPG (red), and gold (green) substrates. 

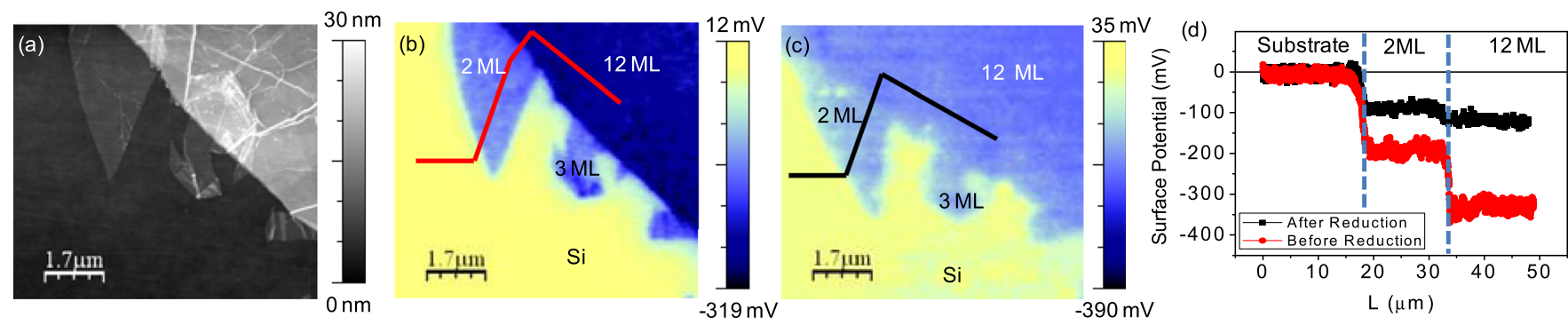

FIG. 2. (a) AFM topographic image of GO layers on a Si substrate. The upper-right region corresponds to a multilayered region with height of 15 nm (i.e., 12 layers). In the central area, we observe a bilayered GO. Panels (b) and (c) are surface potential maps of the same region as panel (a) acquired before and after chemical reduction of the GO layers. The profiles shown in panel (d) correspond to the same path on the surface potential before and after reduction. Here, we observe that the surface potential difference between layers decreases after chemical reduction. The expected saturation behaviour can also be observed for the 12 monolayers layers region.

with increasing number of layers for the reduced samples, and also a lower difference between the substrate and the first rGO layer.

Our results can be understood in terms of charge transfer between the substrates and the GO layers. The lower surface potential on GO layers than the substrates is indicative of electron transfer from the substrates to the $\mathrm{GO}$ and $\mathrm{rGO}$ layers, suggesting a smaller charge transfer at the substraterGO interface than for the more oxidized samples due to the lower presence of oxygen containing groups with high electron affinity. The reported values of surface potentials steps are smaller than what could be expected from the work by $\mathrm{Wu}$ et $a l .{ }^{26} \mathrm{We}$ attribute this difference to two different causes: different level of oxidation/reduction of GO and the anomalous screening effects in GO and RGO; the combination of extreme thinness of the GO layers and high in-plane conductivity with low density of charge carriers makes electric field screening effect in these samples much less effective than in ideal metals. ${ }^{27}$ The fact that electric fields partially penetrate the GO and RGO layers leads to surface potential values not comparable to that of work functions reported in literature. The incomplete screening of electric fields through GO and RGO layers implies that measured surface potential should also have a contribution from the substrate, presenting lower differences than that expected if we consider work function differences. This kind of effects have been already reported in literature for graphene obtained by mechanical exfoliation of graphite ${ }^{18}$ and for that obtained via Silicon Carbide graphitization. ${ }^{17,28}$

As mentioned above, all the measurements reported so far were carried out in high vacuum conditions and elevated sample temperatures $\left(60^{\circ} \mathrm{C}\right)$ with the aim of reducing possible effects of adsorbates and/or a water layer on the surface potential that might hinder access to the intrinsic properties of the thins films (see Figure S2 in SI). In order to evaluate the influence of environmental conditions, we also performed similar experiments at atmospheric pressure with variable ambient humidity and sample temperature. Surface potential maps of the same region under different temperatures and humidity conditions are shown in Figure 3. These images reveal that the surface potential steps observed in vacuum conditions and at low humidity smear out as ambient humidity is increased, suggesting a shielding effect of a thin layer of water on the surface. Our observation that differences of surface potential of about $50 \mathrm{mV}$ are shielded already at relative humidity $(\mathrm{RH})=30 \%$ and $\mathrm{T}=20^{\circ} \mathrm{C}$ is compatible with the effect of water on hydrophilic surfaces. ${ }^{29}$

Finally, we also carried out more conventional electrostatic force microscopy (EFM) measurements, available in almost every commercial basic AFM set up. EFM maps were acquired using a two pass mode, usually known as retrace or lift mode. In this mode, simultaneous topographic and electrostatic interaction maps are obtained. For the samples reported here, this method yields qualitative information (data are shown in SI) similar to KPFM and can be also used as a fast, contactless, and non invasive characterization procedure of the coverage of GO films on different substrates.
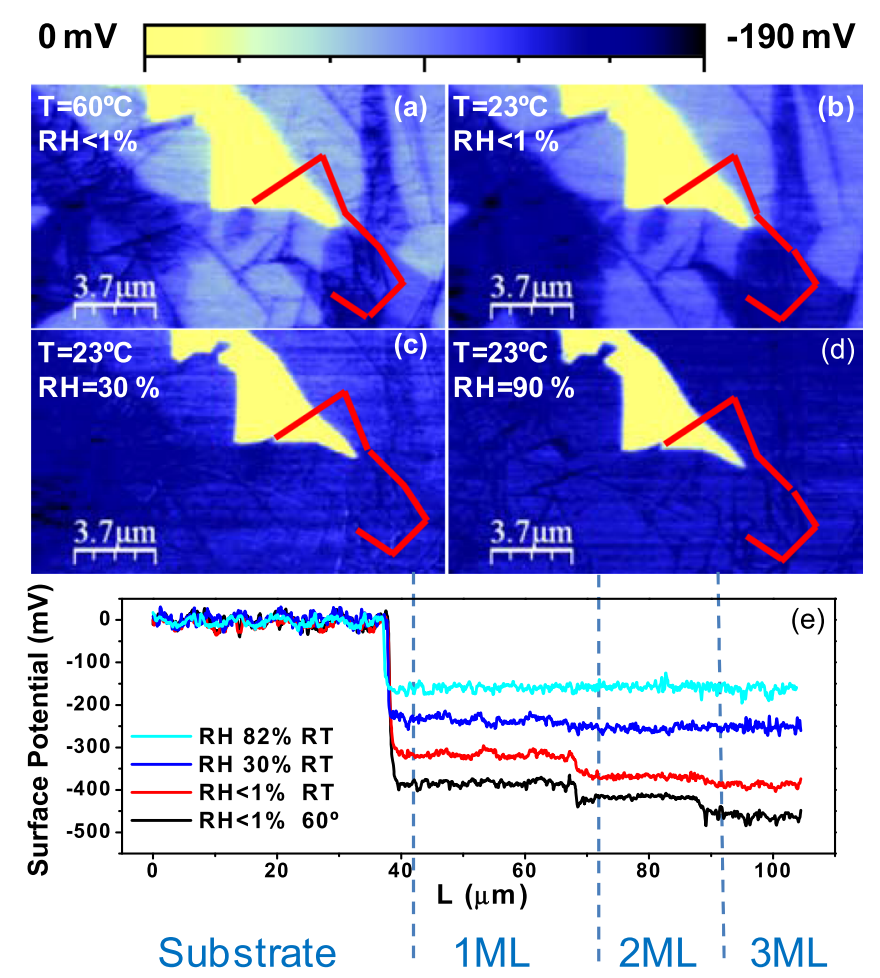

FIG. 3. (a)-(d) KPFM images of the same region of GO deposited on HOPG under different environmental conditions. Sample temperature (T) and environmental $\mathrm{RH}$ are indicated in white in each image. The images are equalized saturating the substrate in order to optimize the visual discrimination of layers when possible. The red line indicates schematically the paths followed to perform the profiles shown in panel (e). The paths followed are the same in the four images. In the profiles depicted in panel (e), we can observe how the surface potential smears out with increasing relative humidity. At $30 \%$, humidity changes in the surface potential can hardly be appreciated. 
In summary, KPFM maps of GO thin films on different substrates demonstrate a coverage dependent surface potential; the surface potential decreases in steps with increasing number of layers until five layers are reached; at this coverage, the surface potential saturates to a constant value. The situation is similar for its conducting counterpart, rGO, but with smaller potential steps between layers. These results evidence electron transfer from metallic-type substrates to GO layers and reveal that singular screening effects are present in few layered graphene oxide thin films. This intrinsic behavior is smeared out in the presence of ambient humidity were the surface potential is shielded by the presence of a thin water layer on the surface. We also demonstrate that this effect can be exploited to quickly determine the number of layers of GO on a substrate with a fast and simple EFM technique. The reported changes in surface potential as well as the effect of environment can help to characterize future device performance in a quick and simple manner.

${ }^{1}$ K. S. Novoselov, A. K. Geim, S. V. Morozov, D. Jiang, Y. Zhang, S. V. Dubonos, I. V. Grigorieva, and A. A. Firsov, Science 306, 666 (2004).

${ }^{2}$ C. Lee, X. D. Wei, J. W. Kysar, and J. Hone, Science 321, 385 (2008).

${ }^{3}$ A. A. Balandin, S. Ghosh, W. Bao, I. Calizo, D. Teweldebrhan, F. Miao, and C. N. Lau, Nano Lett. 8, 902 (2008).

${ }^{4}$ C. R. Dean, A. F. Young, I. Meric, C. Lee, L. Wang, S. Sorgenfrei, K. Watanabe, T. Taniguchi, P. Kim, K. L. Shepard, and J. Hone, Nat. Nanotechnol. 5, 722 (2010).

${ }^{5}$ I. Gierz, C. Riedl, U. Starke, C. R. Ast, and K. Kern, Nano Lett. 8, 4603 (2008).

${ }^{6}$ J. Rafiee, X. Mi, H. Gullapalli, A. V. Thomas, F. Yavari, Y. Shi, P. M. Ajayan, and N. A. Koratkar, Nature Mater. 11, 217 (2012).

${ }^{7}$ K. S. Novoselov, D. Jiang, F. Schedin, T. J. Booth, V. V. Khotkevich, S. V. Morozov, and A. K. Geim, Proc. Natl. Acad. Sci. U.S.A. 102, 10451 (2005).

${ }^{8}$ T. A. Land, T. Michely, R. J. Behm, J. C. Hemminger, and G. Comsa, Surf. Sci. 264, 261 (1992).

${ }^{9}$ C. Berger, Z. M. Song, T. B. Li, X. B. Li, A. Y. Ogbazghi, R. Feng, Z. T. Dai, A. N. Marchenkov, E. H. Conrad, P. N. First, and W. A. de Heer, J. Phys. Chem. B 108, 19912 (2004).
${ }^{10}$ S. Stankovich, D. A. Dikin, G. H. B. Dommett, K. M. Kohlhaas, E. J. Zimney, E. A. Stach, R. D. Piner, S. T. Nguyen, and R. S. Ruoff, Nature 442, 282 (2006); S. Stankovich, R. D. Piner, X. Q. Chen, N. Q. Wu, S. T. Nguyen, and R. S. Ruoff, J. Mater. Chem. 16, 155 (2006).

${ }^{11}$ K. A. Mkhoyan, A. W. Contryman, J. Silcox, D. A. Stewart, G. Eda, C. Mattevi, S. Miller, and M. Chhowalla, Nano Lett. 9, 1058 (2009).

${ }^{12}$ C. Gomez-Navarro, R. T. Weitz, A. M. Bittner, M. Scolari, A. Mews, M. Burghard, and K. Kern, Nano Lett. 7, 3499 (2007).

${ }^{13}$ V. Lopez, R. S. Sundaram, C. Gomez-Navarro, D. Olea, M. Burghard, J. Gomez-Herrero, F. Zamora, and K. Kern, Adv. Mater. 21, 4683 (2009).

${ }^{14}$ G. Eda, G. Fanchini, and M. Chhowalla, Nat. Nanotechnol. 3, 270 (2008).

${ }^{15}$ J. T. Robinson, F. K. Perkins, E. S. Snow, Z. Wei, and P. E. Sheehan, Nano Lett. 8, 3137 (2008).

${ }^{16}$ M. Zhou, Y. Zhai, and S. Dong, Anal. Chem. 81, 5603 (2009).

${ }^{17}$ T. Burnett, R. Yakimova, and O. Kazakova, Nano Lett. 11, 2324 (2011).

${ }^{18}$ S. S. Datta, D. R. Strachan, E. J. Mele, and A. T. C. Johnson, Nano Lett. 9, 7 (2009); N. J. Lee, J. W. Yoo, Y. J. Choi, C. J. Kang, D. Y. Jeon, D. C. Kim, S. Seo, and H. J. Chung, Appl. Phys. Lett. 95, 222107 (2009).

${ }^{19}$ See http://www.nanoinnova.com/ for N.I. Technologies.

${ }^{20}$ W. S. Hummers and R. E. Offeman, J. Am. Chem. Soc. 80, 1339 (1958).

${ }^{21}$ I. Horcas, R. Fernandez, J. M. Gomez-Rodriguez, J. Colchero, J. GomezHerrero, and A. M. Baro, Rev. Sci. Instrum. 78, 013705 (2007).

${ }^{22}$ T. R. Albrecht, P. Grutter, D. Horne, and D. Rugar, J. Appl. Phys. 69, 668 (1991).

${ }^{23}$ M. Jaafar, D. Martinez-Martin, M. Cuenca, J. Melcher, A. Raman, and J. Gomez-Herrero, Beilstein J. Nanotechnol. 3, 336 (2012).

${ }^{24}$ J. M. R. Weaver and D. W. Abraham, J. Vac. Sci. Technol. B 9, 1559 (1991); T. Glatzel, S. Sadewasser, and M. C. Lux-Steiner, Appl. Surf. Sci. 210, 84 (2003); M. Nonnenmacher, M. P. Oboyle, and H. K. Wickramasinghe, Appl. Phys. Lett. 58, 2921 (1991).

${ }^{25}$ R. S. Sundaram, C. Gomez-Navarro, E. J. H. Lee, M. Burghard, and K. Kern, Appl. Phys. Lett. 95, 223507 (2009).

${ }^{26}$ X. S. Wu, M. Sprinkle, X. Li, M. Fan, C. Berger, and W. A. De Heer, Phys. Rev. Lett. 101, 026801 (2008).

${ }^{27}$ C. Gomez-Navarro, F. J. Guzman-Vazquez, J. Gomez-Herrero, J. J. Saenz, and G. M. Sacha, Nanoscale 4, 7231 (2012).

${ }^{28}$ T. Filleter, K. V. Emtsev, T. Seyller, and R. Bennewitz, Appl. Phys. Lett. 93, 133117 (2008).

${ }^{29}$ H. Sugimura, Y. Ishida, K. Hayashi, O. Takai, and N. Nakagiri, Appl. Phys. Lett. 80, 1459 (2002).

${ }^{30}$ See supplementary material at http://dx.doi.org/10.1063/1.4773357 for sample preparation, EFM, and KPFM imaging. 